\title{
Advanced Sensory Electronics and Systems with Self-x Capabilities by MEMS Switch Integration
}

\author{
Muhammad Akmal Johar ${ }^{1}$, Andreas Koenig ${ }^{1}$ \\ ${ }^{1}$ Institute of Integrated Sensor Systems, Technical University of Kaiserslautern, D-67663 \\ Kaiserslautern Germany \\ johar@eit.uni-kl.de, koenig@eit.uni-kl.de
}

\begin{abstract}
:
After entering mass application, more stringent requirements on flexibility, accuracy and dependability are imposed on sensory systems and their electronic embodiment. Analog static and dynamic reconfiguration, culminating in so called self-x features, e.g., self-trimming or -repairing, requires capable switch resources. While other switching solutions in electromechanical relays and CMOS switches have their own advantages and disadvantages, MEMS switches can become an alternative in switching solutions. Our research group focuses on applying and designing MEMS switches to better enable self-x features in 3D AMR smart sensor nodes, e.g., to adapt to ambient changes. The AMR sensor performance has tendency to detoriate over the time when it is used repeatedly and also affected with ambient temperature change. The self-x features will increase the robustness and the adaptability of the sensor node. Two levels of implementation have been demonstrated; at system level and at component level. At system level, MEMS switch has been used to enable self-monitoring and self-repairing features. At component level, AMR sensor from Sensitec and the designated MEMS switches are equipped with self-monitoring and self repairing features. A first PCB -level demonstrator with commercial MEMS-switches has been designed to be used in the localization system context. It also will serve for dedicatedly designed MEMS switches. Initial test results show the MEMS switches offer extra benefits compared to other commercial products. The designated MEMS switches also achieved lower resistance contact $(<3 \Omega)$, lower switching actuation at $70 \mathrm{~V}$ with additional functions of self-monitoring and self-repairing. Future works will bring further effort to combine these functions to single chip integration.
\end{abstract}

Key words: Magneto resistive Sensor, Self-x features, self-configurable, self-repairing, DC MEMS switches

\section{Introduction}

Electronic devices nowadays are equipped with many sensory elements to increase the intelligent level of the devices. Industry standard required high degree of accuracy, robustness and reliability in order to give maximum consumer satisfaction. Several approaches have been introduced to meet this requirement. The current trend in analog electronics is to move from static state circuitry to dynamic reconfiguration of circuitry. The self$x$ features in analog electronics have been introduced e.g., self-trimming, self-monitoring and self-repairing. To enable these functions in analog electronics the capable switches are required.

In general, there are two major types of electronics switches in the market which are electromagnetic relays and CMOS switches. Electromagnetic relays are dominant in the industrial automation devices. They can handle high power application, has low contact resistance and gives total signal separation in off-state. However these devices generally have slower switching response, big in sizes and have relatively high power consumption. Thus electromagnetic relays are generally not suitable for mobile devices of consumer electronics. CMOS switches have advantages of being small in size, can reach high switching speed and low power consumption. However the drawbacks are it has high resistance during on-state, small leakage current during off-state and relatively can handle only low power signal. CMOS switches low degree of isolation of signal line.

This market scenario gives opportunity for new types of switches using MEMS technology. MEMS switches offer the benefits of the both types of switches and reduce the weaknesses 
as formerly discussed. MEMS switches are small in size, have total signal isolation, low in power consumption and higher power handling compared to CMOS. Recent market survey [1] estimated a significant increase of MEMS switch demand in coming years to replace the conventional switching components in the industrial automation products such as Automated Test Equipment (ATE). The components replacement from electromagnetic relays to MEMS switches will lead to smaller and mobile ATE products. Manufacturing industries will get a direct benefit from this change as it will increase the flexibility of testing equipment setup and thus further reduced the production cost.

The possibility of MEMS switches to replace CMOS circuit also has been investigated and reported [2, 3]. This research group has shown some exciting results and benefits in using MEMS switches in digital circuits.

On the other hand our research group vision is to build reconfigurable sensory electronics system. We are looking at the possibility in implementing MEMS switches to enable reconfigurable and dynamic analog electric system. Specific requirements for this application have been identified e.g. moderate switching frequency, low contact resistance and operating in DC signal domain.

As for now, industries already embrace MEMS switches for high frequency application in RF switches. These types of MEMS switches have moveable structures without any physical contact within and that make it as a switch element suitable only at high frequency only. It is easier to reach the industries standard in terms of reliabilities and product lifetime. However in order to handle DC signal, MEMS switches facing additional challenge. DC switch requires physical contact during switching actions and that exposes the contact area with tear and wear problem. The real challenge is to design the MEMS switch that can withstand the rigorous demand of contact actions. Another potential issue that MEMS device posses is the stiction phenomenon. This phenomenon happens when MEMS structures are stick together randomly. It can happen during releasing process during manufacturing causing low production output yield or during operating time which can cause hazard to the users. Currently in the market, there is only one product line of MEMS switches still available for DC signal applications offered by Radant MEMS [4].

\section{AMR Smart Sensor Nodes and Self-x Features}

The research group of ISE has involved in designing and developing the AMR wireless sensor node for indoor localization. The overall research vision is to have a smart sensor node that is reliable, robust and adaptable with the changes of the environment. The reliability of the sensor is in the sense of minimal error in measurement. In this project, the magnetic field has been used as a medium to detect sensor nodes position in an industrial tank. The industrial tanks will be equipped with a controllable magnetic field produce by several coils. The sensor node is equipped with three axes ( $x, y$ and $z$ ) of AMR sensors for localization purposes $[5,6]$ via detecting changes of magnetic field from the coils.

As AMR sensors provide the ability to localize the sensor node positions, they have small drawback that may reduce their performances. AMR sensors show degradation effects when used repetitively. This effect has been demonstrated and reported in the previous publications [7]. The sensor also can turn into saturated condition when exposed to high magnetic field. In this situation, the sensor can be considered 'blind' as it cannot detect the changes magnetic field anymore. AMR sensor also shows degradation effect towards the changes of ambient temperature. As the ambient temperature rises, the sensor produces significant measurement error.

In order to improve the AMR sensors performances, several solutions have been suggested and implemented as shown in Fig. 1. An ideal solution will be a solution that using minimum number of component, does not adding the complexity of the system and easy to implement. The notions of self-x capabilities attracted the researcher interest lately as a way adding the system intelligent.

A single axis of AMR sensor node has been built to demonstrate the implementation of self$x$ features using MEMS switches. This demonstrator as shown in the Fig. 2 is built from discrete components that have DC-DC converter, DC MEMS switches, CMOS switches, instrumental amplifying circuit and AMR sensor.

There are several self- $x$ systems that have been introduced and implemented in this sensor node. They have been implemented in two levels; at system level and at component level. 


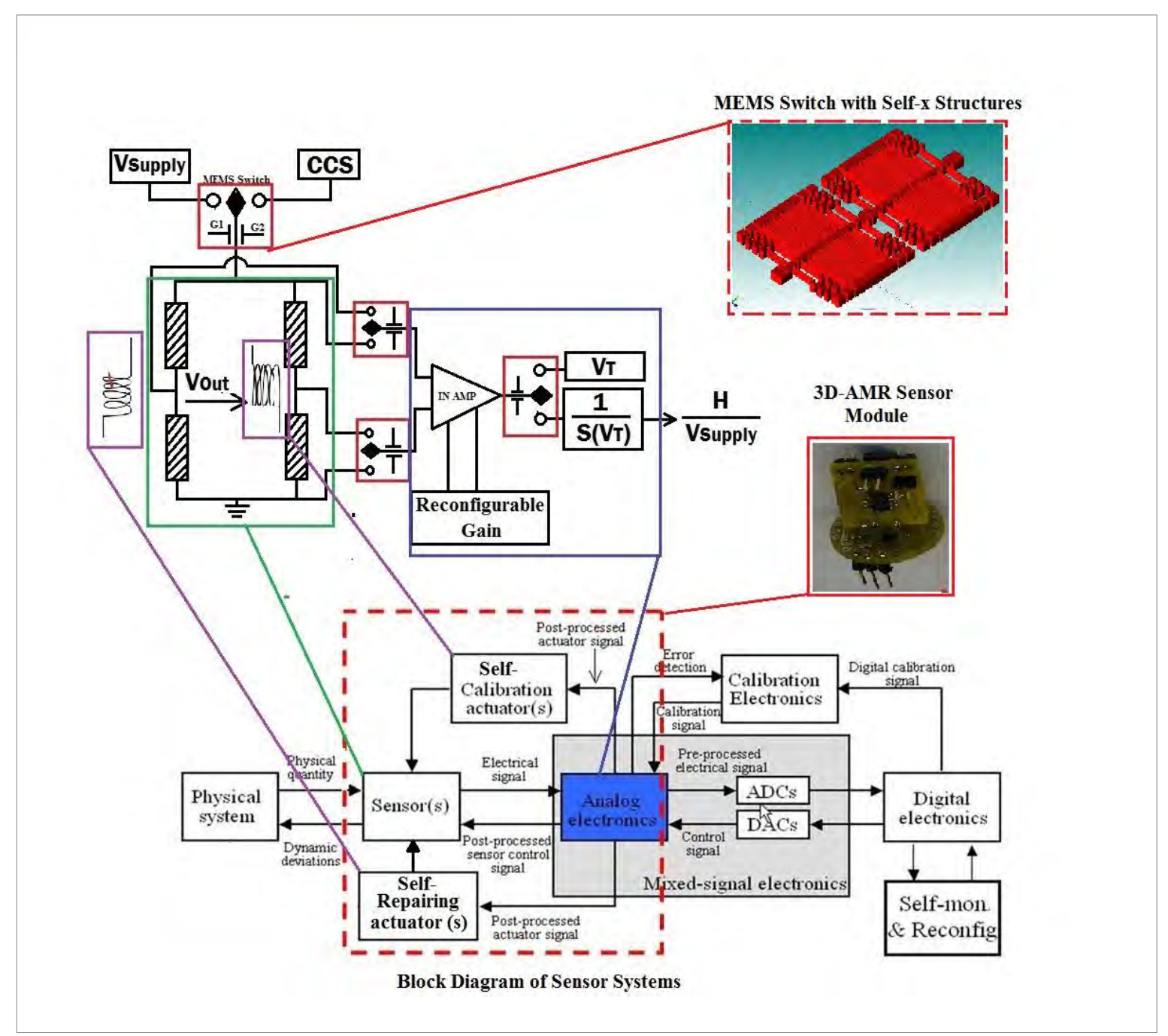

Fig.1 Block diagram of AMR sensor node with self-x features implementation.

Self-monitoring has been implemented in the system level to monitor the ambient temperature with minimum effort as shown in Fig. 2 (A). A smart temperature measurement system has been proposed and reported [8]. The same AMR sensor bridge has been used to measure magnetic field and ambient temperature. In this application, low contact resistance is not a critical requirement so the CMOS switch is used due to the limited sources of MEMS switches.

Another implementation at the system level is self-trimming of the signal gain as shown in Fig. 2 (B). This is required when the AMR sensor start to show deterioration of the magnetic field measurement thus required gain adjustment at the instrumentation amplifier. The demonstrator is using INA 122 instrumentation amplifier. This amplifier allows gain setting by changing the value of gain resistor $\left(R_{G}\right)$. Here the MEMS switches have been used to switch the desired resistance value to obtain the desired amplifier gain. The low contact resistance of the switches and small form factor made it as a superior choice compared to CMOS switches that have relatively high contact resistance at around $90 \Omega$ during switch on state. There will be no off-set resistance adjustment required which are also temperature dependent as in the CMOS switches. The more precise resistance value can be achieved for gain trimming in the sensor signal.

On the other hand, the implementation of self- $x$ features at the component level has been done at several components. In this sensor node, AMR sensor AFF755B from Sensitec has been used [9].This sensor has been equipped with two internal coils that enable self-monitoring and self-repairing features. The first coil is used to monitor the state of the AMR sensor by creating a small magnetic field from a small current across the coil. This controllable field will give information that the sensor not in the saturation state. If the sensor could not read the expected known magnetic field, the controller 
will notice that the sensor may in the saturation mode.

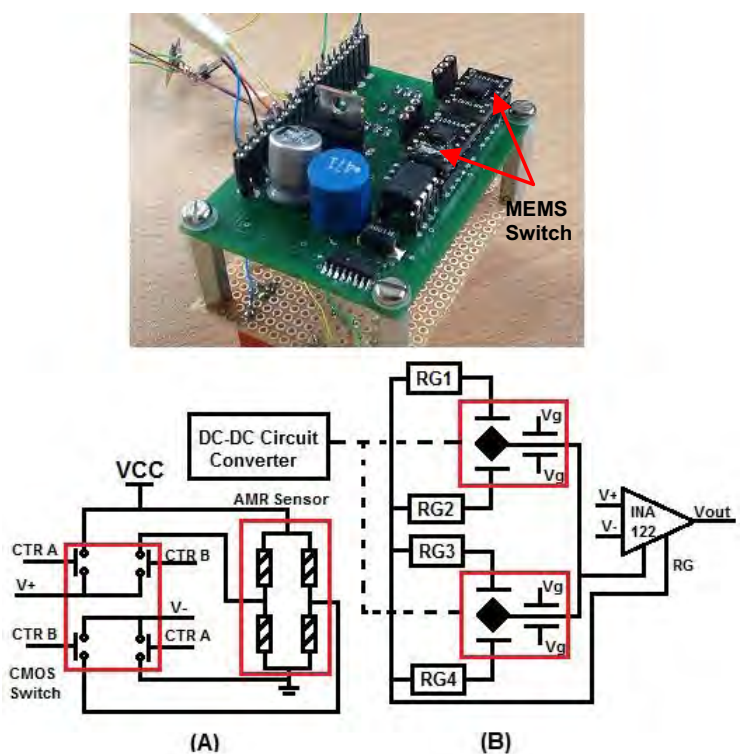

Fig. 2. Single axis AMR sensor node with self-x features implementation

The main reason that turned the sensor into saturation state is due to the presence of external magnetic field that strong enough to change the arrangement of magnetic domain in the sensor bridges changes. In order to recover the AMR sensor capability, the self-repairing mode needs to be activated. This can be done by using the second coil inside the AMR sensor. This coil will produce a strong magnetic field that able to rearrange the magnetic domain in the sensor bridge back to the original position. This action will repair the sensor back to their optimum functionality.

\section{MEMS Switch Design and Evaluation Results}

In the previous section, the self- $x$ features implementations have been discussed. The advantages of using MEMS switches and the place where it have been used also have been detailed. This section will look into more detailed on the MEMS switch itself.

In the sensor node test bed, the MEMS switches RMSW220HP produced by Radant MEMS have been used. The switch is in the QFN form factor that allows easy integration with the test bed PCB. It requires $90 \mathrm{~V}$ of electrostatic actuation voltage to activate it. Electrostatic actuation consumed very small power as less than $10 \mu \mathrm{A}$ is required in the $\mathrm{DC}$ DC voltage converter MAX774 from maxim. The type of the switch is Single Pole Double Throw (SPDT) switch. It can operate from DC to $8 \mathrm{GHz}$ of switching speed. This makes it as a suitable candidate for switching the reconfigurable analog circuit. The test result shows this switch able to perform switching functions as expected. There is no interference in measurement as the switch has very low contact resistance $(<4 \Omega)$.

In parallel with that, this research group is also put a lot of effort in designing the MEMS switches with additional self-x features inside. It is the main intention to extend the application of self-x features at component level in MEMS switches. As the current MEMS switch in the market still lack of these additional smart features, the in-house MEMS switch design is equipped with additional structures to provide self-monitoring and self repairing actions.

The MEMS switches are build using MetalMUMPs technology offered by MEMSCAPE Inc. It has two moveable bodies mass that make connections during switching actions and closing the contacts. The contacts are covered by gold layer thus provide low contact resistance. The switches are mainly driven by electrostatic actuators at each body mass. Each body mass have two sets of heat actuators that also act as the anchors. Each heat actuator set has the opposite movement from each other. These provide closing and opening switch actions when it is required.

The switch design is so flexible in terms of the usage. There are several mode that can be choose depends on the resources and limitations of the circuit applications. For typical mobile devices, where the power sources are limited, electrostatic actuator as primary mover is favorable. The heat actuator will be the selfrepairing element to remedy the stiction problem. Self-monitoring can be implemented by using the structure at the end of the body mass via capacitance difference measurement. A further detailed concerning the switch design and simulation result has been reported [10].

For industrial applications such as Automated Test Equipment (ATE) where power is not a constraint, heat actuators can be used as primary mass mover. This is because a higher force can be produce by these actuators thus a better closed contact can be achieved. The electrostatic comb actuator can be turned into self-monitoring structures with greater sensitivity compared to the previous one.

The Karl Suss PM5 wafer probe and Keithley 804 high power supply unit have been used for measurement as shown in Fig 3. Initial test sets include determining the functionality of the actuators, the contact resistance of the switch, confirming the functions self- $x$ features. 


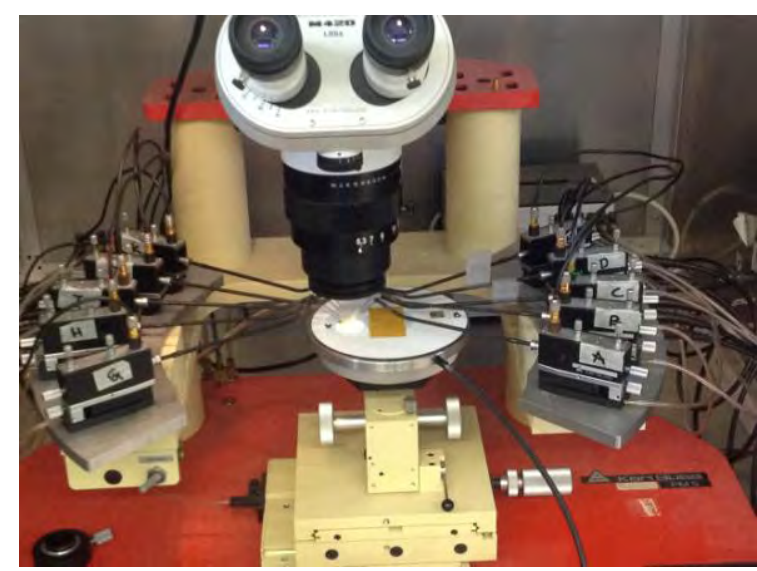

Fig. 3. Wafer probe (R) setup for MEMS evaluation

The initial evaluation result of MEMS switch prototype show promising result of the functions of self-x features. The MEMS switch has low contact resistance $(<3 \Omega)$. However this is a tentative pessimistic value because the influence of the measurement setup has not been compensated. There are two types of switches that have been designed and developed namely; SPST- Single Pole and Single Throw (Fig. 4) and DPDT- Double pole and Double Throw (Fig. 5). The SPST switch has two body masses and can handle one signal. As for the DPDT switch contain three bodies mass system and can handle two different signals.

Both set of heat actuators are moving in the correct direction when activated. That means one set of heat actuators (label as Heat Actuator A) can be use for closing the switch when the current flows through it. The switch will return to open state once the current supply is cut. The current required for this action are around $100 \mathrm{~mA}$ to $150 \mathrm{~mA}$ depending on the switch design. The other set of heat actuator (label as Heat Actuator B) produced an opposite direction movement which is useful for self-repairing features. Experiment result shows a short current pulse of $50 \mathrm{~ms}$ with $50 \mathrm{~mA}$ is enough to release stiction that occurred in the comb structures. Each pulse has $7.5 \mathrm{~mW}$ of power consumption and use $0.1875 \mathrm{~mJ}$ of energy.

On the other hand, the initial results electrostatic actuators have shown the needs of design improvement. The overall actuator size of $570 \times 800 \mu \mathrm{m}$ (width $x$ finger length) required the actuation voltage of around $70 \mathrm{~V}$. This is $20 \mathrm{~V}$ less if compared to the commercial products. While this might not be significant in the current setup where the discrete component is used, it will be significant when the DC-DC converter charge pump design is design monolithically together with other analog electronic circuits. However the electrostatic actuators did not show the same consistency as heat actuators. The stiction phenomenon is bound to happen after several runs of actuations. However this problem are proved temporary as a self-repairing action via heat actuator can released the stiction by applying small current across them.

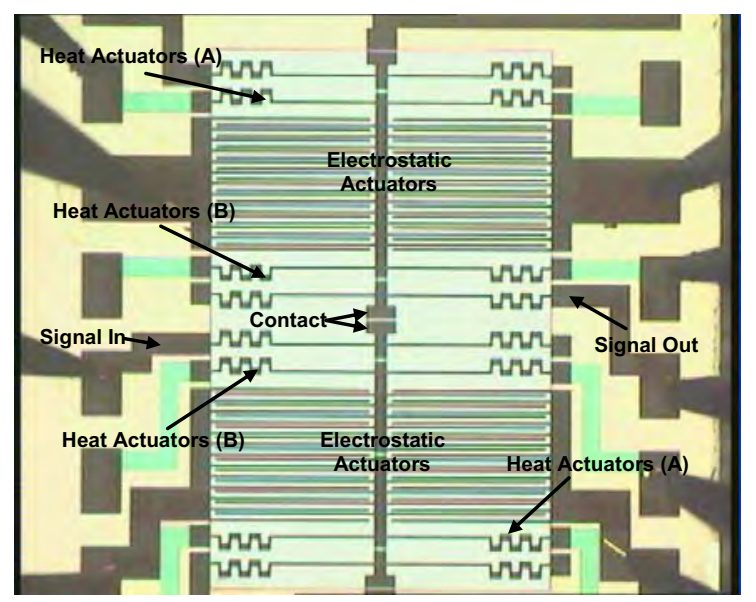

Fig. 4. Screen-shot of SPST MEMS switch

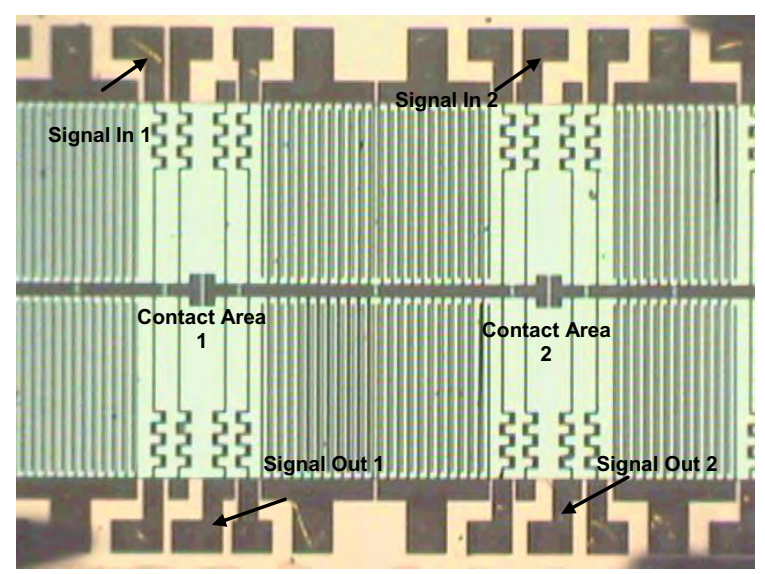

Fig. 5. Screen-shot of DPDT MEMS switch

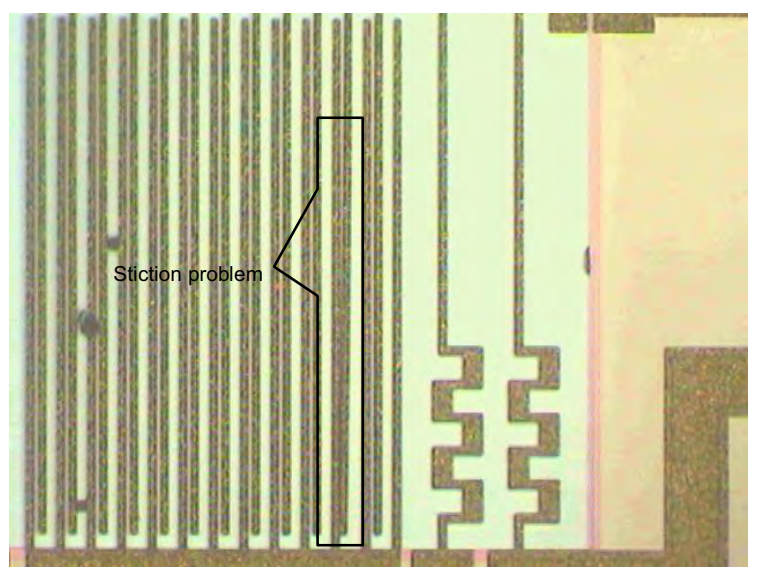

Fig. 6. Stiction phenomena that occurred in the comb structure

There are several possible reasons on why electrostatic actuators are not functioning really 
well. The prototype of MEMS switches come in dies form without packaging and tested using wafer probe under room environment. Thus the electrostatic actuators need to overcome an additional air resistance in order to move. A better performance can be expected when an appropriate packaging on the MEMS die are implemented. The air resistance can be neglected then as the condition inside the package are near vacuum. The other factor is the sizing of the finger length which might be too long and make it easier to attract a single finger sticking with other finger. Design improvement studies are required here to find the optimum size of electrostatic actuators without affecting too much the overall switch size. As summary, the comparisons between these two MEMS switches are shown in Tab. 1.

Tab. 1: Comparison between two different MEMS switch

\begin{tabular}{|c|c|c|}
\hline Properties & Radant MEMS & ISE MEMS \\
\hline $\begin{array}{c}\text { Switch } \\
\text { Movement }\end{array}$ & $\begin{array}{c}1.4 \mathrm{~mm} \times \\
1.45 \mathrm{~mm}\end{array}$ & $\begin{array}{c}2.69 \mathrm{~mm} \times \\
1.91 \mathrm{~mm}\end{array}$ \\
\hline Actuator & Electrostatic & $\begin{array}{c}\text { Electrostatic } \\
\text { or/ and Heat }\end{array}$ \\
\hline $\begin{array}{c}\text { Contact } \\
\text { resistance }\end{array}$ & $<4 \Omega$ & $<3 \Omega^{*}$ \\
\hline $\begin{array}{c}\text { Actuation } \\
\text { Voltage }\end{array}$ & $90 \mathrm{~V}$ & $70 \mathrm{~V}$ \\
\hline $\begin{array}{c}\text { Self-x } \\
\text { features }\end{array}$ & No & Yes \\
\hline
\end{tabular}

\section{Conclusion and Future Works}

Based on the PCB level prototype, the implementation of self- $x$ features has been successfully adapted. The sensor node has a certain degree of intelligence and robustness level to adapt with the changes in surrounding while maintaining the optimum performance of measurement. The MEMS switches are proved to be better choices compared to the other counterpart as lower contact resistance minimize the error in AMR sensors without giving any offset in measurement.

Further characterization test on the current MEMS prototype are still actively done. The behavior of the switch and other switch properties will be reported in the near future.

Future works will concentrate on the scaling properties of the MEMS switches. The scaling properties are important as it will provide the information to optimize MEMS design. The optimum size of electrostatic actuators that produced low actuation voltage, low tendencies of stiction phenomenon and small in size are desired. As for the heat actuator the size that requires lower power consumption without sacrificing the switch operation bandwidth are desired.

Apart from that, we are also looking at the possibility to replace the discrete component and bring the functions circuits to the integrated circuit level. Several dies from different integrated circuit technologies can be combine under one package; namely AMS H35 High Voltage CMOS technology for DC-DC converter circuits, MEMS die and normal CMOS for analogue circuits. This will produce a more compact design of sensor node and increase the mobility of the product.

\section{References}

[1] Williamson, J., Automated Test Equipment: An Overview of MEMS and non-MEMS Switching Options, in MEMS Investor Journal. 2011.

[2] Hei, K., et al., Design, Optimization, and Scaling of MEM Relays for Ultra-Low-Power Digital Logic. Electron Devices, IEEE Transactions on, 2011. 58(1): p. 236-250.

[3] Nathanael, R., et al. 4-terminal relay technology for complementary logic. in Electron Devices Meeting (IEDM), 2009 IEEE International. 2009.

[4] RMSW220HP-QFN: SPDT, High Power Broadband RF MEMS Switch (DC-8GHz). 2003, Radant MEMS. p. 1-3.

[5] S. Carella, K.I., A. Koenig. 3D-Localization of Low-Power Wireless Sensor Nodes Based on AMR Sensors in Industrial and Aml Applications in 15ITG-/GMA- F- Sens und Messyst. 2010.

[6] Koenig, A., et al. Dynamically Reconfigurable Integrated Sensor Electronics for Magnetic Localisation of Distributed Autonomous Sensor Nodes in AMA Conferences Sensor 2013 Nurnberg, Germany.

[7] Johar, M.A. and A. Koenig, Case Study of an Intelligent AMR Sensor System with Self-x Properties, in Soft Computing in Industrial Applications, T. Gaspar-Cunha, Ricardo, G. Schaefer, and L. Costa, Editors. 2010, Springer Berlin Heidelberg. p. 337-346.

[8] Johar, M.A., R. Freier, and A. Koenig. Adding self-x capabilities to AMR sensors as a first step towards dependable embedded systems. in Intelligent Solutions in Embedded Systems (WISES), 2011 Proceedings of the Ninth Workshop on. 2011. Regensburg Germany.

[9] AFF755B AMR Sensor Data sheet. 2011, Sensitec $\mathrm{GmbH}$. p. 6.

[10] Johar, M.A. and A. Koenig. DC MEMS Switches with Self-x Features: Design, Simulation and Implementation Strategies. in 10th IEEE International Conference on Semiconductor Electronics [ICSE 2012]. 2012. Kuala Lumpur. 